\title{
Anwendung von Apremilast bei moderater bis schwerer atopischer Dermatitis bei Kindern
}

\author{
Rachael C Saporito David Cohen \\ Division of Dermatology, Department of Internal Medicine, Mercer University School of Medicine, Macon, GA, USA
}

Schlüsselwörter

Atopische Dermatitis · Atopisches Ekzem · Atopie .

Pädiatrische Dermatologie · Apremilast

\section{Zusammenfassung}

Die atopische Dermatitis (AD) ist eine chronische, pruritische Hauterkrankung, die häufig durch eine bakterielle Superinfektion erschwert wird und von der 10,7\% der US-amerikanischen Kinder betroffen sind. Zur Pathogenese gehört ein Zusammenbruch der Hautbarriere neben einer Dysfunktionalität sowohl der angeborenen als auch der adaptiven Immunantwort, einschließlich einer unausgewogenen Zunahme von T-Helferzellen vom Typ 2 (Th2Zellen) und einer Hyperimmunglobulinämie E. Die erhöhte Anzahl an Th2-Zellen führt durch die Freisetzung von Interleukin (IL)-4, IL-5 und IL-13 zur Produktion von Immunglobulin E und zur Eosinophilie und durch die Freisetzung von IL-10 zu einer Verminderung des Schutzes vor einer bakteriellen Superinfektion. Die gegenwärtig von der Food and Drug Administration (FDA) zugelassene sympto- matische Behandlung der AD umfasst topische Salben, topische und systemische Kortikosteroide, topische immunmodulierende Therapien, Antibiotika sowie Phototherapie. Es gibt jedoch keine zugelassenen gezielten Therapien oder Heilmethoden. Der hier vorgestellte Fallbericht eines 8-jährigen afroamerikanischen Jungen unterstützt eine neuartige Therapie der moderaten bis schweren AD mit Apremilast, einem Phosphodiesterase-Typ-4-Inhibitor. Kürzlich wurde eine klinische Phase-II-Studie (NCT02087943) zu Apremilast für die Behandlung von AD bei Erwachsenen abgeschlossen. Dieser Fallbericht veranschaulicht das Potenzial von Apremilast als AD-Therapie für Kinder, bei denen ein großer Bedarf an sicheren und wirksamen Medikationen besteht.

C 2017 S. Karger GmbH, Freiburg

\section{Einleitung}

Die atopische Dermatitis (AD) ist eine chronische, pruritische Hauterkrankung, die häufig durch eine bakterielle Superinfektion erschwert wird und von der 10,7\% der US-amerikanischen Kinder betroffen sind [1]. Zur Pathogenese gehört ein Zusammenbruch der Hautbarriere neben einer Dysfunktionalität sowohl der angeborenen als auch der adaptiven Immunantwort, einschließlich einer unausgewogenen Zunahme von T-Helferzellen vom Typ 2 (Th2-Zellen) und einer Hyperimmunglobulinämie E [2]. Die erhöhte Anzahl an Th2-Zellen führt durch die Freisetzung von Interleukin (IL)-4, IL-5 und IL-13 zur Produktion von Immunglobulin E (IgE) und zur Eosinophilie und durch die Freisetzung von IL-10 zu einer Verminderung des Schutzes vor einer bakteriellen
Superinfektion. Die gegenwärtig von der Food and Drug Administration (FDA) zugelassene symptomatische Behandlung der AD umfasst topische Salben, topische und systemische Kortikosteroide, topische immunmodulierende Therapien, Antibiotika sowie Phototherapie. Es gibt jedoch keine von der FDA zugelassenen gezielten Therapien oder Heilmethoden [2]. Viele Ärzte gehen zur Off-Label-Anwendung systemischer, immunmodulierender Therapien über, z.B. mit Ciclosporin, Mycophenolat-Mofetil, Methotrexat und Azathioprin. Es besteht ein großer ungedeckter Bedarf an sicheren Medikamenten zur Behandlung der schweren, persistierenden $\mathrm{AD}$, besonders bei Kindern.

Apremilast ist ein Phosphodiesterase-Typ-4 (PDE4)-Inhibitor, der zur Behandlung der moderaten bis schweren Psoriasis vom Plaque-Typ und der Psoriasis-Arthritis indiziert ist [3]. Kürzlich

\section{KARGER}

Fax +4976145207 14

information@karger.com

www.karger.com

\section{(c) 2017 S. Karger GmbH, Freiburg}

Accessible online at:

www.karger.com/kkd
Rachael Saporito

308 Hospital Drive, Suite 200

Macon, GA 31217, USA

saporito_rc@med.mercer.edu 
Tab. 1. Diagnosekriterien für atopische Dermatitis

\begin{tabular}{lc}
\hline American Academy of Dermatology [13] & Kriterien von Hanifin und Rajka [14] \\
\hline $\begin{array}{l}\text { Wesentliche Merkmale (müssen vorhanden sein) } \\
\text { Pruritus }\end{array}$ & Wichtige Kriterien (hiervon müssen 3 vorhanden sein) \\
Ekzem (akut, subakut, chronisch) & Pruritus \\
Typische Morphologie und alters- & Dermatitis auf flexuralen Oberflächen bei Erwachsenen oder im Gesicht und \\
spezifische Mustera & auf Extensorflächen bei Kleinkindern \\
Chronische oder rezidivierende Anamnese & Chronische oder rezidivierende Dermatitis \\
Wichtige Merkmale & Persönliche oder Familienanamnese mit kutaner oder respiratorischer Allergie \\
Frühes Alter des Krankheitsbeginns & Sekundäre Kriterien (hiervon müssen 3 vorhanden sein) \\
Atopie & Gesichtsmerkmale (infraorbitale Verdunklung etc.) \\
Persönliche und/oder Familienanamnese & Auslöser (Umweltfaktoren etc.) \\
lgE-Reaktivität & Komplikationen (Neigung zu Hautinfektionen etc.) \\
Xerosis & Sonstiges (frühes Alter des Krankheitsbeginns, trockene Haut etc.)
\end{tabular}

a Zu den Mustern gehören: Betroffenheit von Gesicht, Hals und Extensoren bei Kleinkindern und Kindern; aktuelle oder frühere flexurale Läsionen in jeder Altersgruppe, wobei Leisten- und Achselgegend nicht betroffen sind.

wurde eine klinische Phase-II-Studie (NCT02087943) zu Apremilast zur Behandlung der AD bei Erwachsenen abgeschlossen. Es wurde gezeigt, dass Patienten mit AD im Vergleich zu Kontrollen eine erhöhte Phosphodiesterase-Aktivität in ihren Leukozyten aufwiesen, was einen wichtigen Entzündungsmechanismus bei AD darstellt [4-7]. Wir beschreiben hier ein Kind mit schwerer AD, bei dem herkömmliche Therapien versagt hatten, das aber auf eine Apremilast-Therapie gut ansprach.

\section{Fallbericht}

Ein 8-jähriger afroamerikanischer Junge mit Asthma sowie Umwelt- und Nahrungsmittelallergien in der Anamnese erschien zu einem Nachsorgetermin beim Dermatologen. Er wies einen persistierenden Ausschlag an den oberen und unteren Extremitäten sowie an Rumpf und Hals und auf der Kopfhaut auf. Es handelte sich um einen pruritischen, lichenifizierten Ausschlag mit Krustenbildung, der bereits seit Jahren in schwerwiegender Form bestand, trotz der im Folgenden beschriebenen Eskalation der Therapie durch den Dermatologen. Zwei Jahre zuvor, als der Junge zum ersten Mal beim Arzt erschien, durchlief er eine von seinem Allergologen angeordnete Therapie mit Tacrolimus-Salbe und topischer Desonid-Creme, die nur eine minimale Besserung der Läsionen und des Pruritus bewirkte. Zu seinen Labordaten gehörte ein IgE-Spiegel von 11,769 IE/ml (Normalbereich 0-90 IE/ml) und ein relativ erhöhter Anteil von Eosinophilen an den Leukozyten von $8,1 \%$ (Normalbereich $0-7 \%$ ), die mit $0,44 \times 10^{3} / \mu \mathrm{l}$ jedoch absolut normal vertreten waren (Normalbereich $0,0-0,5 \times$ $\left.10^{3} / \mu \mathrm{l}\right)$. Der Patient war laut Anamnese familiär vorbelastet, da bei beiden Eltern im Kindesalter eine AD diagnostiziert worden war. $\mathrm{Zu}$ diesem Zeitpunkt stellte der Dermatologe bei dem Kind die klinische Diagnose einer AD aufgrund seiner erythematösen, ekzematösen Flecken und der Lichenifizierung, die 60\% der Körperoberfläche des Jungen bedeckte (Tab. 1). Es wurden keine Biopsie durchgeführt, da diese zur Diagnose einer AD nicht empfohlen wird. Beim ersten Termin wies der Patient ferner eine ho-

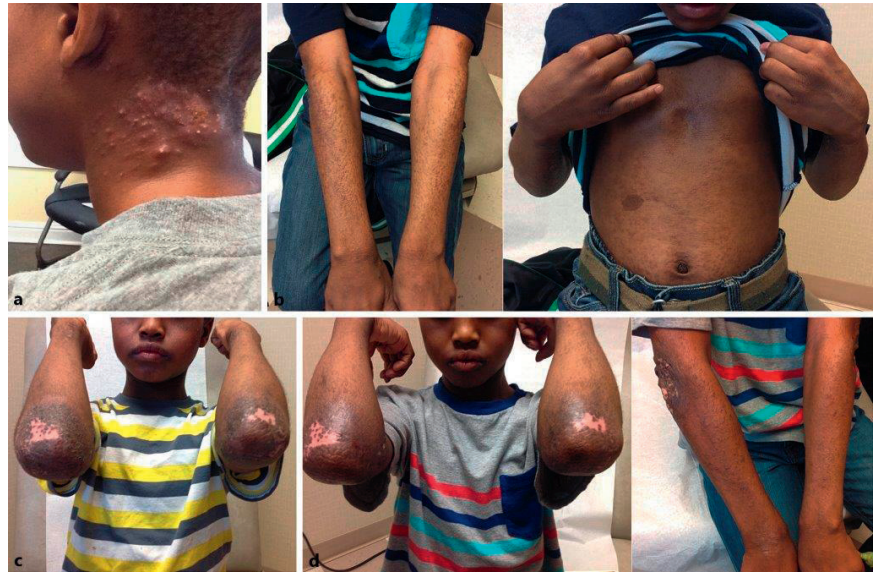

Abb. 1. Klinisches Erscheinungsbild vor und während der ApremilastTherapie. a Halsabszesse bei der Vorstellung im Alter von 6 Jahren. b Patient nach der Behandlung mit topischen Immunsuppressiva und systemischen Kortikosteroiden. c Patient vor der Apremilast-Behandlung. d Patient 8 Wochen nach Verwendung von Apremilast.

nigfarbene Verkrustung, die auf eine bakterielle Superinfektion hindeutete, und Abszesse am Hals auf (Abb. 1a). Ihm wurde für 2 Wochen Trimethoprim-Sulfamethoxazol verschrieben. Angesichts dieser Infektionsanzeichen wurde eine systemische Immunsuppression zur Eindämmung der AD bis zum Abklingen der Infektion aufgeschoben. Er wurde 2-mal täglich mit topischer Triamcinolonacetonid-Salbe $(0,1 \%)$ behandelt und bekam die Anweisung, weiterhin Desonid im Gesicht, in der Leistengegend und in der Achselhöhle zu verwenden. Zwei Monate später, als die Infektion abgeklungen war, wurde eine aggressivere Behandlung verschrieben, die Prednison $20 \mathrm{mg}$, Myophenolat-Mofetil 200 $\mathrm{mg} / \mathrm{ml}$ topisch für den Körper und topisches Tacrolimus für das Gesicht beinhaltete.

Auch 7 Monate nach Eskalation der Behandlung mit Immunsuppressiva waren $50 \%$ der Körperoberfläche noch immer mit demselben stark pruritischen Ausschlag bedeckt (Abb. 1b). Man 


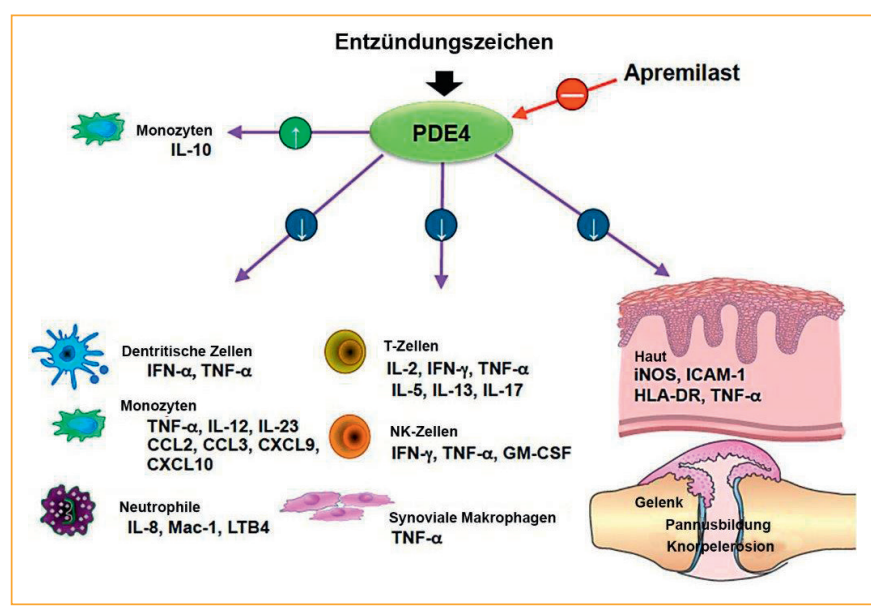

Abb. 2. Die Hemmung der PDE4 durch Apremilast wirkt sich auf Leukozyten und Keratinozyten aus, indem es die Freisetzung von Entzündungsmediatoren moduliert, die vermutlich die Entzündungsreaktion bei atopischer Dermatitis auslösen. Abbildung mit freundlicher Genehmigung der Celgene Corporation.

begann bei dem Patienten nun mit Injektionen von Omalizumab 300 mg gegen Asthma alle 2 Wochen, in der Hoffnung, dass sich durch diese Injektionen auch die anderen atopischen Erkrankungen des Patienten, darunter die AD, bessern würden. Fünf Monate nach diesen Injektionen wurde dem Patienten - im Alter von 8 Jahren - eine Off-Label-Behandlung mit Apremilast 30 mg täglich verschrieben (der Hälfte der Erwachsenendosis von $30 \mathrm{mg}$ 2-mal täglich), weil seine Haut nach wie vor Exkoriationen, Hyperpigmentierung und starken Pruritus aufwies (Abb. 1c). Apremilast bewirkte eine rasche und nachhaltige Linderung des starken Pruritus in nur 2 Wochen, ohne Nebenwirkungen. Das Haar des Patienten begann wieder über die Läsionen der Kopfhaut zu wachsen, und die Läsionen des übrigen Körpers wiesen signifikant weniger Entzündungen und Exkoriationen auf (Abb. 1d).

\section{Diskussion}

Zyklonukleotid-Phosphodiesterasen umfassen eine Gruppe von 11 Isoenzymen, die die Second Messenger cAMP (zyklisches Adenosinmonophosphat) und cGMP (zyklisches Guanosinmonophosphat) zu inaktiven Molekülen abbauen und den jeweiligen intrazellulären Spiegel senken. Speziell bei AD bewirkt die vermehrte Phosphodiesterase-Aktivität einen proinflammatorischen Zustand, indem sie für eine weitere Reduzierung zyklischer Nukleotide sorgt [4-8]. Abnorm niedrige Spiegel zyklischer Nukleotide wiederum können zu erhöhten Spiegeln von Prostaglandin $E_{2}\left(\mathrm{PGE}_{2}\right)$ führen, wodurch Interferon- $\gamma($ IFN- $\gamma)$ reduziert wird und es außerdem zu einer Erhöhung von IL-4 kommen kann, das Th2-Zellen anregt $[9,10]$. Th2-Zellen setzen IL-4, IL-5 und IL-13 frei, die daran beteiligt sind, die IgE-Produktion anzuregen und eine Eosinophilie hervorzurufen. Man nimmt an, dass die Freisetzung des entzündungshemmenden Zytokins IL-10, das auch an der Th2-Immunantwort beteiligt ist, die Häufigkeit bakterieller Superinfektionen von AD-Läsionen erhöht.
Der genaue Wirkungsmechanismus von Apremilast bei AD-Patienten ist noch unbekannt. Möglicherweise kann jedoch die Hyperaktivität des Immunsystems bei AD durch eine Hemmung der Phosphodiesterase-Aktivitätserhöhung wieder auf den Grundzustand abgesenkt werden [4]. Apremilast hemmt speziell die PDE4, die in Keratinozyten und verschiedenen Leukozyten des angeborenen und adaptiven Immunsystems zu finden ist, darunter Langerhans-Zellen, dendritische Zellen, Monozyten, T-Helferzellen und Eosinophile $[4,8,11]$. Die Hemmung der PDE4 erhöht den cAMP-Spiegel in Leukozyten, wodurch sich die Anzahl der proinflammatorischen Zytokine verringert und die Aktivität eines entzündungshemmenden Zytokins, IL-10, erhöht [4, 8]. In-vivound In-vitro-Studien haben gezeigt, dass Apremilast, indem es auf eine Vielzahl verschiedener Zellen einwirkt, die Konzentrationen wichtiger Mediatoren von Entzündungsreaktionen moduliert (Abb. 2). Apremilast reduziert die Freisetzung von IL-12 und IL-23 aus Monozyten, die damit normalerweise die Th1- bzw. Th17-Zellen anregen $[4,11,12]$. Ferner vermindert es den $\mathrm{PGE}_{2}-$ Spiegel, wodurch die Th2-Zellantwort abnehmen kann. Dadurch werden die von T-Helferzellen freigesetzten Entzündungsmediatoren unterdrückt, darunter IFN- $\gamma$ und Tumornekrosefaktor- $\alpha$ (TNF- $\alpha$ ) aus Th1-Zellen, IL-4 und IL-13 aus Th2-Zellen sowie IL-17 und IL-22 aus Th17-Zellen [11]. Diese Zytokine - insbesondere IL-4 und IL-17 - regen im weiteren Verlauf Keratinozyten zur Produktion von IL-31 an, wodurch der Pruritus ausgelöst wird [12]. Durch die Verringerung der Th2- und Th17-Immunantwort nimmt daher auch der Pruritus ab.

Der Mehrwert von Apremilast gegenüber den gegenwärtig erhältlichen oralen Medikamenten zur Behandlung einer moderaten bis schweren AD besteht in seiner sicheren Verwendbarkeit, wobei Durchfall, Übelkeit, Infektionen der oberen Atemwege und Kopfschmerzen die am häufigsten dokumentierten Nebenwirkungen sind, während Endorganschäden unbekannt sind $[4,12]$. Zudem besserten sich der Pruritus und die Lebensqualität durch den Einsatz bei AD wesentlich, wie auch schon in klinischen Studien zur Behandlung von Psoriasis mit Apremilast gezeigt wurde [4]. Ein weiteres Beispiel eines Biologikums für eine ähnlich gezielte $\mathrm{AD}$-Behandlung ist Dupilumab, ein monoklonaler Antikörper, der an die $\alpha$-Untereinheit des IL-4-Rezeptors bindet und die Förderung der Th2-Antwort durch IL-4 und IL-13 hemmt [12]. In einer klinischen Phase-III-Studie (NCT02277769) zeigt Dupilumab gegenwärtig das Potenzial, die erste von der FDA zugelassene gezielte Therapie zur Behandlung der Symptome einer moderaten bis schweren $\mathrm{AD}$ bei Erwachsenen zu werden [12]. Ziel bei der Entwicklung gezielterer Therapien ist es, die Nebenwirkungen bei der Behandlung einer moderaten bis schweren $\mathrm{AD}$ zu vermindern sowie die Therapietreue und -wirksamkeit zu erhöhen.

\section{Schlussfolgerung}

$\mathrm{AD}$ ist eine chronische, unheilbare Erkrankung, für die es bei moderaten bis schweren Krankheitsformen bisher nur ineffektive von der FDA zugelassene Therapien gibt. Ärzte benötigen sichere 
Behandlungsmöglichkeiten, um die AD-Symptome besonders bei Kindern zu lindern. Durch künftige klinische Studien und Feedback von Dermatologen, die die pädiatrische Patientengruppe behandeln, könnte sich Apremilast als effektives Medikament zur Linderung des starken Pruritus und der Entzündung von Hautläsionen erweisen.

\section{Ethikerklärung}

Der Erziehungsberechtigte des Kindes hat eine Einwilligungserklärung abgegeben.

\section{Disclosure Statement}

R.S. hat keine relevanten Beziehungen mit der Branche offenzulegen. D.C. ist Berater, Sprecher und klinischer Forscher für die Celgene Corporation.

\section{Literatur}

1 Shaw TE, Currie GP, Koudelka CW, Simpson EL: Eczema prevalence in the United States: data from the 2003 National Survey of Children's Health. J Invest Dermatol 2011;131:67-73.

2 Leung TN, Hon KL: Eczema therapeutics in children: what do the clinical trials say? Hong Kong Med J 2015;21:251-260.

3 Abdulrahim $\mathrm{H}$, Thistleton S, Adebajo AO, Shaw T, Edwards C, Wells A: Apremilast: a PDE4 inhibitor for the treatment of psoriatic arthritis. Expert Opin Pharmacother 2015;16: 1099-1108.

4 Samrao A, Berry TM, Goreshi R, Simpson EL: A pilot atudy of an oral phosphodiesterase inhibitor (apremilast) for atopic dermatitis in adults. Arch Dermatol 2012;148:890-897.

5 Grewe SR, Chan SC, Hanifin JM: Elevated leukocyte cyclic AMP-phosphodiesterase in atopic disease: a possible mechanism for cyclic AMP-agonist hyporesponsiveness. J Allergy Clin Immunol 1982;70:452-457.
6 Chan SC, Reifsnyder D, Beavo JA, Hanifin JM: Immunochemical characterization of the distinct monocyte cyclic AMP-phosphodiesterase from patients with atopic dermatitis. J Allergy Clin Immunol 1993;91:1179-1188.

7 Hanifin JM, Chan SC: Monocyte phosphodiesterase abnormalities and dysregulation of lymphocyte function in atopic dermatitis. J Invest Dermatol 1995; 105(suppl):84S-88S.

8 Moustafa F, Feldman SR: A review of phosphodiesterase-inhibition and the potential role for phosphodiesterase 4-inhibitors in clinical dermatology. Dermatol Online J 2014;20:22608.

9 Adams M, Horan G, Schafer PH: 24th European Academy of Dermatology and Venereology Congress; 7-11 October; Copenhagen, Denmark.

10 Chan SC, Kim JW, Henderson WR Jr, Hanifin JM: Altered prostaglandin E2 regulation of cytokine production in atopic dermatitis. J Immunol 1993;151:3345-3352.
11 Schafer PH, Parton A, Gandhi AK, et al: Apremilast, a cAMP phosphodiesterase- 4 inhibitor, demonstrates anti-inflammatory activity in vitro and in a model of psoriasis. Br J Pharmacol 2010;159:842-855.

12 Notaro ER, Sidbury R: Systemic agents for severe atopic dermatitis in children. Paediatr Drugs 2015;17:449-457.

13 Eichenfield LF, Tom WL, Chamlin SL, et al: Guidelines of care for the management of atopic dermatitis: section 1. Diagnosis and assessment of atopic dermatitis. J Am Acad Dermatol 2014;70:338-351.

14 Hanifin JM, Rajka G: Diagnostic features of atopic dermatitis. Acta Derm Venereol Suppl (Stockh) 1980;92:44-47. 